\title{
Research on the Optimal Selection for Dams Construction Sites Based on the Power Flow Calculation
}

\author{
Guangjin SHEN \\ Department of Electrical Engineering and Automation, North China Electric Power University, \\ Baoding, 071000, China \\ email: 18331136782@163.com
}

Keywords: The Kariba Dam, Power Flow Calculation, Optimal Selection, Multivariate Analysis

\begin{abstract}
In this paper, the location optimization scheme based on stepwise screening method is put forward to assist the Zambezi River management. Firstly, we evaluate three options based on multivariate analysis and find that the third option is the most appropriate one. Then we analyze with improved Analytic Hierarchy Process to filter out a series of unsuitable dams' construction sites. And we use power flow calculation in electric system to solve the dams' security and water supply problem. The method is further improved in providing the guidance for extreme water flows. It is shown that our model has important practice guiding significance in managing the Zambezi River.
\end{abstract}

\section{Introduction}

Southern Africa's longest trans-boundary river, the Zambezi, has captured the world's intensive attention owing to The Kariba Dam with controversial construction. The double curvature concrete arch dam, standing 128 meters tall and 579 meters long, forms Lake Kariba which extends for 280 kilometers and holds 185 cubic kilometers of water [1]. According to the report issued by the Institute of Risk Management of South Africa in 2015, the dam is in dire need of maintenance.

In this problem, numerous studies contribute a lot to the question we are analyzing here. However, these researches are limited in that, some of them only put forward removing small dam to improve the problem [2] and some of them just optimized the existed dam without solving the problem fundamentally [3], in the aspect of removing the Kariba Dam and replacing it with a series of smaller dams along the Zambezi River, it still needs further investigation.

We set up an evaluation system based on multivariate analysis to assess the given three options and found that the third option, i.e., removing the Kariba Dam and replacing it with a series of dams, is the optimal one. Provided the effective strategy as swiftly as possible, it is pretty significant for us to decompose the problem into the following three sub-problems, on the basis of our discussion.

How to eliminate some small dams out of position from multitudinous dams owing to water management preliminarily?

How to keep the balance between safety and costs by modulating the water flow through our multiple dam system?

How to embrace exogenous events in consider of extreme conditions listed in the requirements?

In terms of the first sub-problem, we construct a model and do analytic hierarchy process out of routine and then we can filter out unsuitable points. For the second, in order to do security analysis, we analogize power flow calculation in electric system to water flow calculation to make sure dams security. For the last, we transform programming problem into seeking extreme of discrete function by binary code conversion. On the basis of above, we compare Rose Scale Diagram of schemes to get the optimal solution.

\section{Select Potential Dams’ Sites}

We intend to establish dams in positions with high river drops but narrow outlets owing to the high requirement of dam project. After we drawing on the experience of geographical environment conditions of dam construction and simulating the full Zambezi Basin to reconnoiter the terrain 
through Google Earth, fifteen proposed dams' construction sites are preliminary determined. The specific distribution of dams is shown in the topological graph on Fig. 1.

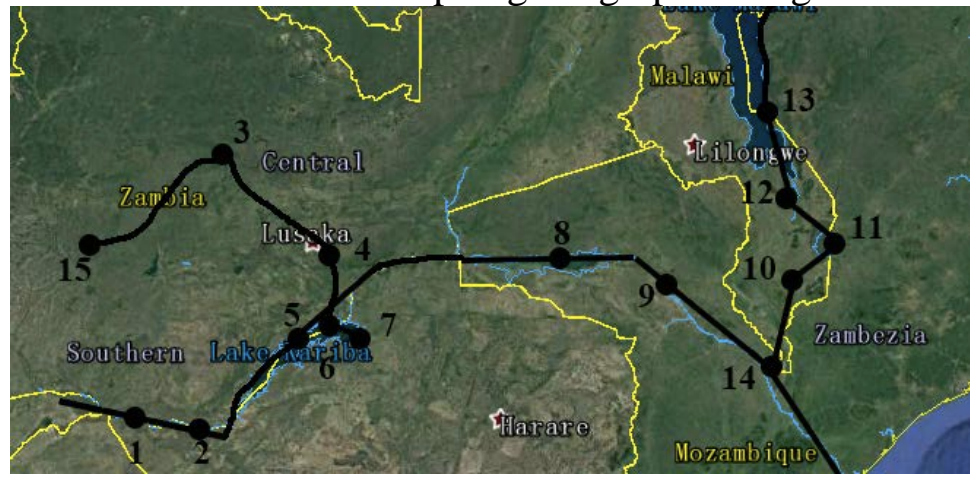

Fig.1 Topological Graph of Points

After determining initial dams' construction sites, we are involved in a heated discussion on water management capabilities of smaller dams including water right, water quality, water ecosystem and so on. Then we select four indexes, namely precipitation, water storage, irrigation amount and hydropower plant generated energy, to conduct a preliminary evaluation of fifteen dams construction sites by means of analytic hierarchy process (AHP), which is shown as Fig.2. Meanwhile, we innovate in AHP. A few addresses of pros and cons are acquired by AHP, and then some low-ranking of them are filtered out due to subjective weigh. As for others, there is no strict standard of advantage and disadvantage between them, and we apply dynamic programming with limits to find the optimal solution.

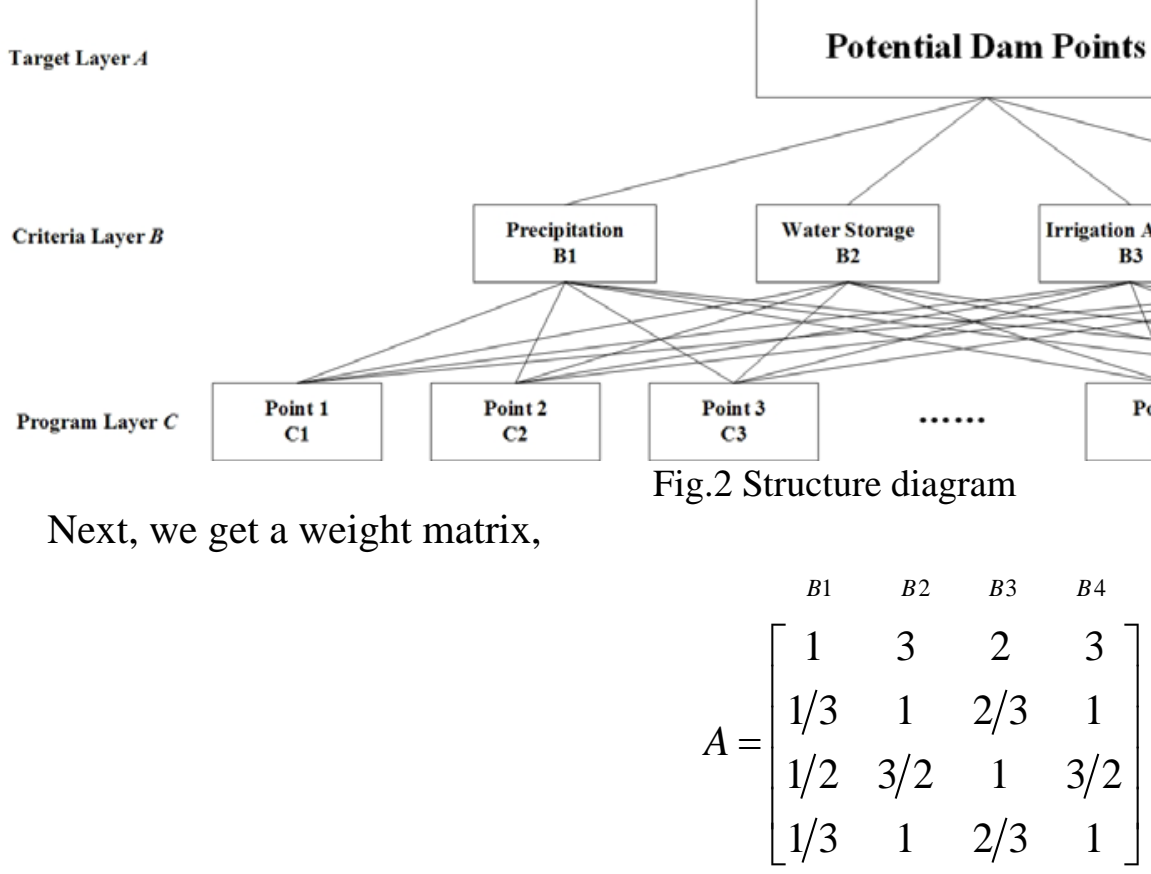

Then, we solve the matrix $A$ and get eigenvector $\omega_{1}$

$$
\omega_{1}=\left[\begin{array}{llll}
0.9748 & 0.8242 & 0.2409 & 0.7871
\end{array}\right]^{T}
$$

And we get corresponding coincidence indicator $R I_{1}$ and $C I_{1}$

$$
\begin{gathered}
R I_{1}=0.90 \\
C I_{1}=\frac{\lambda_{\max }-4}{4-1} \approx 0.037
\end{gathered}
$$

Thus, we calculate

$$
C R_{1}=\frac{C I_{1}}{R I_{1}}=0.041<0.1
$$

So $\omega_{1}$ can be regarded as the weight vector of Layer $C$ to Layer $A$.

In the next step, four items in Criteria Layer will be analyzed, and before it, all variables will be 
unitization processed to emphasize the relationship between new smaller dams and the Kariba dam.

In our model, some relevant parameters of Kariba Dam are regarded as reference value, additionally, we formulate water storage [4] in detail in our next model and collect references about precipitation [5], irrigation amount [6] and hydropower plant generated energy [4]. Thus we can get a primitive matrix $B$.

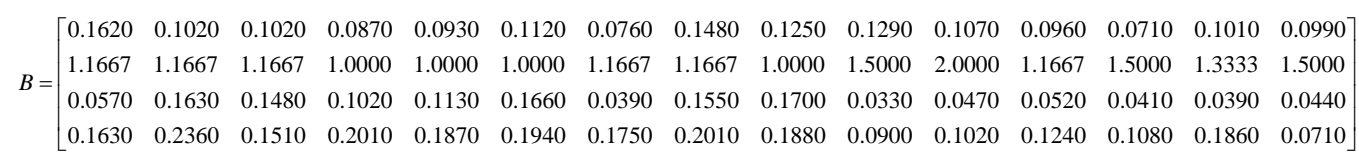

And then, we work out a naturalization calculation value $\omega_{2}$

$$
\begin{aligned}
& {\left[\begin{array}{lllllllllllllll}
0.3715 & 0.4896 & 0.5505 & 0.0390 & 0.4666 & 0.4666 & 0.2248 & 0.2248 & 0.0727 & 0.0727 & 0.0010 & 0.0010 & 0.1321 & 0.1321 & 0.1532
\end{array}\right]^{T}} \\
& \omega_{2}=\left[\begin{array}{lllllllllllllll}
0.2348 & 0.0827 & 0.0827 & 0.0549 & 0.0207 & 0.0207 & 0.7955 & 0.7120 & 0.1338 & 0.0429 & 0.1098 & 0.1087 & 0.0001 & 0.1745 & 0.1753 \\
0.1390 & 0.1576 & 0.1576 & 0.1688 & 0.1688 & 0.0208 & 0.0208 & 0.0208 & 0.0395 & 0.2400 & 0.2400 & 0.4333 & 0.0902 & 0.0902 & 0.0732
\end{array}\right. \\
& \omega_{2}=\left[\begin{array}{lllllllllllllllll}
0.2348 & 0.1390 & 0.1576 & 0.1576 & 0.1688 & 0.1688 & 0.0208 & 0.0208 & 0.0208 & 0.0395 & 0.2400 & 0.2400 & 0.4333 & 0.0902 & 0.0902 & 0.0732 \\
0.2547 & 0.5370 & 0.2409 & 0.2409 & 0.3861 & 0.1753 & 0.2375 & 0.2375 & 0.0660 & 0.0660 & 0.1505 & 0.1505 & 0.2054 & 0.2054 & 0.2394
\end{array}\right] \\
& {\left[\begin{array}{lllllllllllllll}
0.2547 & 0.5370 & 0.2409 & 0.2409 & 0.3861 & 0.1753 & 0.2375 & 0.2375 & 0.0660 & 0.0660 & 0.1505 & 0.1505 & 0.2054 & 0.2054 & 0.2394
\end{array}\right]}
\end{aligned}
$$

Then we work out $C R, \omega$ and $C R_{2}$.

$$
\begin{aligned}
& C R_{2}=\sum_{n=1}^{4} C R_{2}^{(n)}=0 \\
& \omega=\omega_{1} \cdot \omega_{2}=\left[\begin{array}{lllllllllllllll}
0.872 & 0.893 & 0.818 & 0.831 & 0.745 & 0.727 & 0.610 & 0.827 & 0.789 & 0.749 & 0.724 & 0.607 & 0.701 & 0.726 & 0.719
\end{array}\right] \\
& C R=C R_{2}+C R_{1}=0.041<0.1
\end{aligned}
$$

Above all, combination weight $\omega$ can be decision basis.

In conclusion, we get the order of fifteen construction sites. And in this model, we only filter out three less sophisticated points, and then, select the optimal combination from the rest of points. So, we filter out point 7, point 12 and point 15 in the first stage.

\section{Optimal Selection for Dams Construction Sites:}

According to topological graph (Fig.3) in the UAHP model, possible construction sites of dams are picked through model 1 , and then we maintain their original numbers regarding them as nodes $\left(A_{i}\right)$ in Zambezi River basin. In addition, the distance of two nodes is known, we regard injection flow rate of each nose as $Q_{i}$ and node height as $H_{i}$.

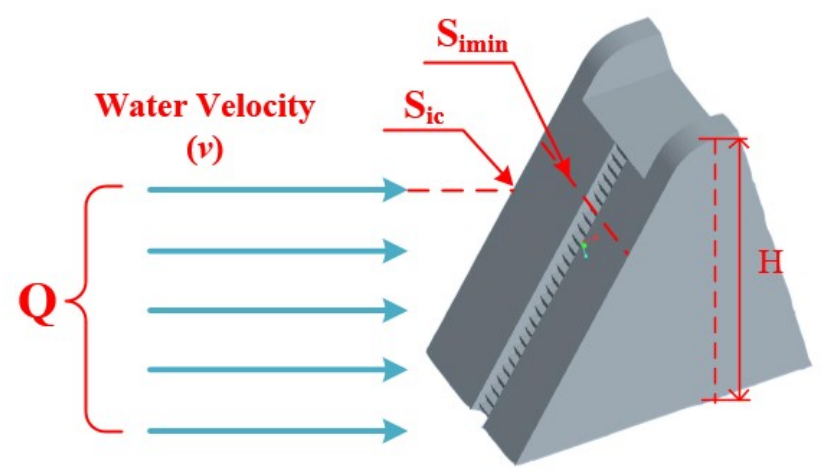

Fig. 3 Analysis of Dams’ Node

On the basis of Bernoulli Equation [7],

$$
P+\frac{1}{2} \rho v^{2}+\rho g H=c
$$

Thus, we conduct that,

$$
v=\sqrt{\frac{2(c-\rho g H-P)}{\rho}}
$$

Combining water specific movement with the function relationship of $v$ and $P$, we can get an equation further.

$$
v=k P^{0.5}
$$

And then, we define proportionality coefficient $k$ as water conductance $Y$ that means, 


$$
Y_{i}=\frac{\sqrt{\frac{2\left(c-\rho g H_{i}-P_{i}\right)}{\rho}}}{P_{i}^{0.5}}
$$

It can be seen that water resistance has only a connection with distance between two nodes, meanwhile water conductance express a reciprocal relationship to water resistance. So we can deduce that water conductance is the reciprocal of distance between two nodes. And $Y_{i i}$ means the sum of all water conductance which are connected with node $A i, Y_{i j}$ means the opposite number of the sum of all water conductance which are connected with node $A_{i}$ and $A_{j}$. Thus we establish water conductance matrix $Y$.

$Y=\left[\begin{array}{ccccccccccccc}0.011494 & -0.01149 & 0 & 0 & 0 & 0 & 0 & 0 & 0 & 0 & 0 & 0 \\ -0.01149 & 0.000778 & 0 & 0 & -0.00253 & -0.0024 & 0 & 0 & 0 & 0 & 0 & 0 \\ 0 & 0 & 0.004785 & -0.00478 & 0 & 0 & 0 & 0 & 0 & 0 & 0 & 0 \\ 0 & 0 & -0.00478 & 0.003559 & -0.01389 & 0 & 0 & 0 & 0 & 0 & 0 & 0 \\ 0 & -0.00253 & 0 & -0.01389 & 0.001087 & -0.04 & -0.00253 & 0 & 0 & 0 & 0 & 0 \\ 0 & -0.0024 & 0 & 0 & -0.04 & 0.002105 & 0 & 0 & 0 & 0 & 0 & 0 \\ 0 & 0 & 0 & 0 & -0.00253 & 0 & 0.001908 & -0.00781 & 0 & 0 & 0 & 0 \\ 0 & 0 & 0 & 0 & 0 & 0 & -0.00781 & 0.002198 & 0 & 0 & 0 & -0.00306 \\ 0 & 0 & 0 & 0 & 0 & 0 & 0 & 0 & 0.004202 & -0.00926 & 0 & -0.00769 \\ 0 & 0 & 0 & 0 & 0 & 0 & 0 & 0 & -0.00926 & 0.00463 & 0 & 0 \\ 0 & 0 & 0 & 0 & 0 & 0 & 0 & 0 & 0 & 0 & 0.005376 & 0 \\ 0 & 0 & 0 & 0 & 0 & 0 & 0 & -0.00306 & -0.00769 & 0 & 0 & 0.001842\end{array}\right]$

Combining with expression of water flow [8],

$$
Q=\mu B\left(\frac{2 P}{\rho}\right)^{0.5}
$$

And then we instead of discharge coefficient with flow velocity $v$, the equation can be conducted,

$$
Q=v B\left(\frac{2 P}{\rho}\right)^{0.5}
$$

We consider that the average pressure of node is $U=P^{0.5}$, and according to equation (13) and equation (17), we can get following equations.

$$
\begin{gathered}
v=Y U \\
Q=\sqrt{\frac{2}{\rho}} B v U
\end{gathered}
$$

At last, we calculate that

$$
\frac{Q_{i}}{\sqrt{\frac{2}{\rho}} B_{i} U_{i}}=\sum_{j=1}^{n} Y_{i j} U_{j}
$$

Based on co-relational studies [4], we know that the water flow of Zambezi River $Q_{\text {total }}$ and $V_{0}$ are functions of time, so we establish correction equation on basis of Newton-Raphson method [9].

$$
\begin{aligned}
& \Delta Q=J \Delta U
\end{aligned}
$$

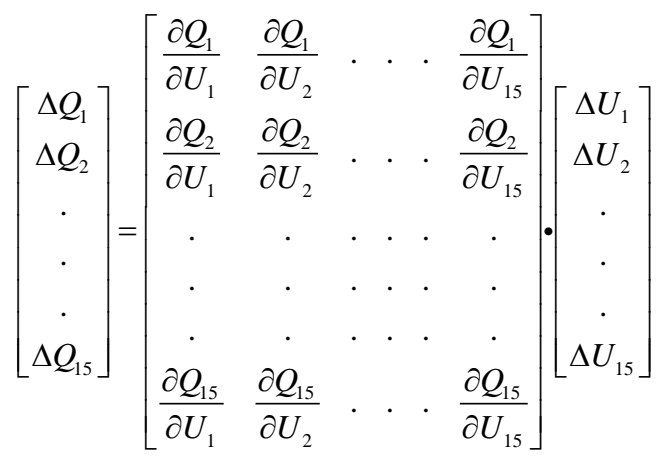




\section{Results and Analysis}

The initial values are as follows. And then doing iteration by Newton-Raphson method, we work out flow rate of each node accurately. Next, we integrate the flow rate and time to get water storage of each node $\left(S_{i}\right)$ in a certain node during a period of time.

$$
S_{i}=\int \sqrt{\frac{2}{\rho}} B_{i} U_{i} \sum_{j=1}^{n} Y_{i j} U_{j} d t
$$

Iterating ten times until reach the pre-assigned precision, we get the following conclusion shown as Fig. 4.

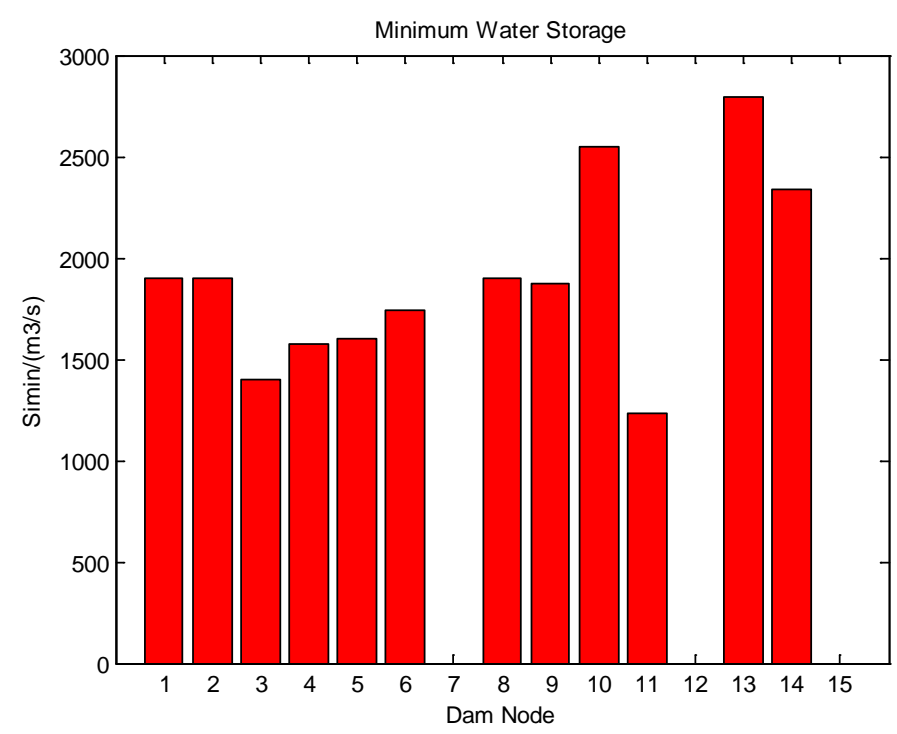

Fig. 4 the Histogram of Minimum Water Storage

While selecting the dams' construction sites, we select some points close to Kariba Dam, among which, point 5 are less distinct, so we can do comparative analysis of other points' relative sizes on the basis of point 5 . And we put $i=5$ in the equation (23), we immediately get a new equation.

$$
S_{5}=\int \sqrt{\frac{2}{\rho}} B_{5} U_{5} \sum_{j=1}^{n} Y_{5 j} U_{j} d t
$$

Next, equation (23) divided by equation (24) is equation (25).

$$
S^{\prime}=\frac{\int B_{i} U_{i} \sum_{j=1}^{n} Y_{i j} U_{j} d t}{\int B_{5} U_{5} \sum_{j=1}^{n} Y_{5 j} U_{j} d t}
$$

And we get normalized water storage of each point on basis of equation (25).

Furthermore, we do data research to find up precipitation and water flow at the flood peak in Zambezi River basin that means critical value $S i_{c}$, which is shown as Fig. 5.

Thus, we reach a conclusion that while minimum water storage of each dam $S i_{\min }>S i_{c}$, the dam we built meets security requirement, in addition, the greater the difference value, the higher the security, however, while $S i_{\min }<S i_{c}$, the dam we built has some potential security risks, and now we should adjust water flow of each node to further adjust water storage of each node and make it in a security rage. In order to obviously reflect the regulating ability, we choose relevant value to measure.

In this model, we gauge the security of dams by comparing maximum volatilities of dams in normal water flow in order to do a further security analysis to dams. Combining equation (16) and equation (23) and then deforming, we figure out the function between water flow and water storage. Put average water flow in, we work out the average water storage $\left(S i_{c}\right)$. In the next step, we compare the extreme that the flow deviates from the average with $S i_{c}$ to figure out a certain dam's volatility. 


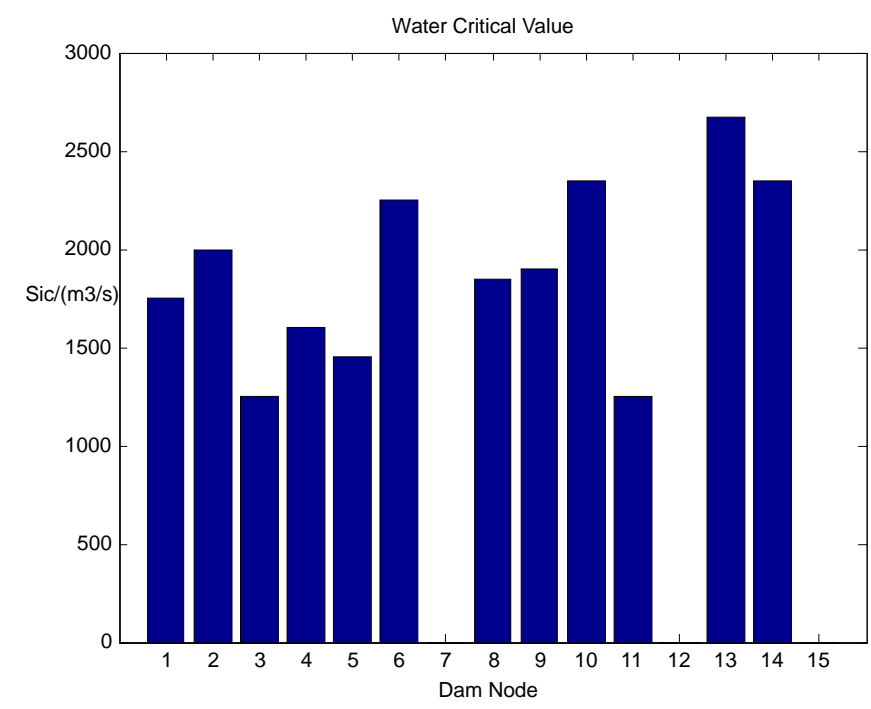

Fig. 5 the Histogram of Water Critical Value

When dams meet security requirements, we further adjust dams' water storages and themselves, i $\mathrm{n}$ consideration of cost and benefit, to figure out the optimal solution. The comparison is shown in Fig. 6

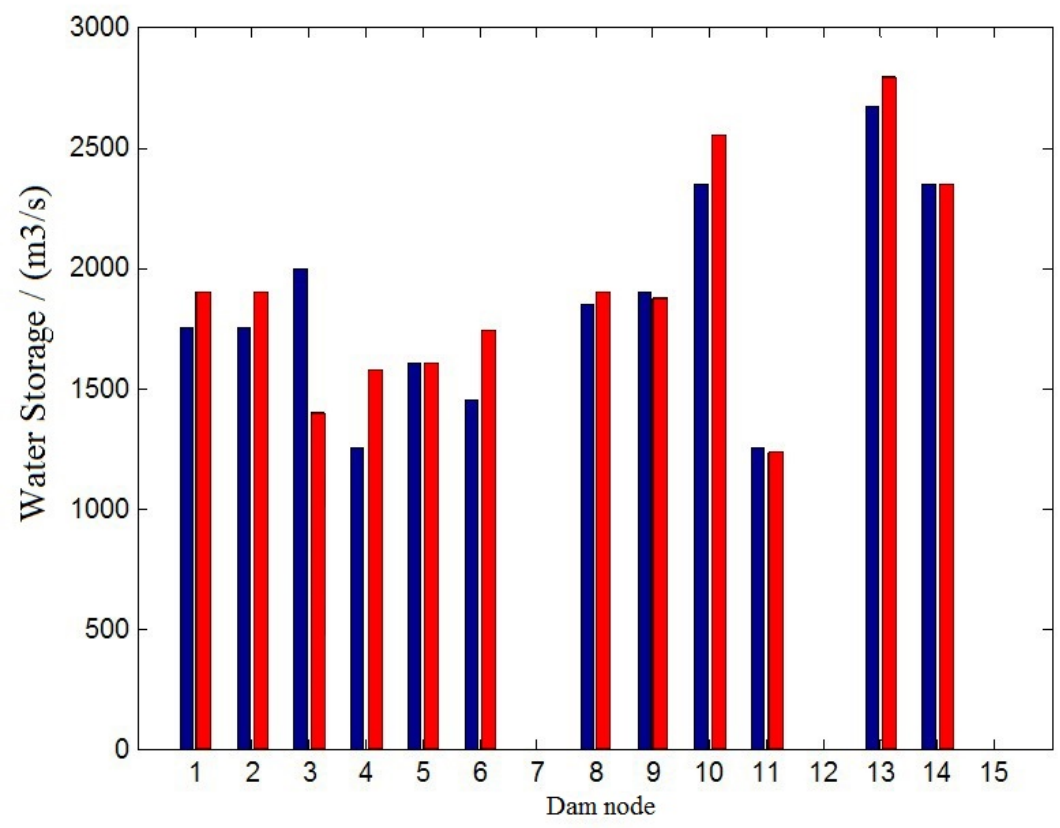

Fig. 6 the Histogram of Comparison

\section{Conclusion}

Through AHP and programming problem changed into extremum problem in discrete mathematics, comprehensively considering safety, capability of water supply, maximum water storage and cost benefit, we get an optimal scheme of location decision of dams. During the process of analyzing, we set up a flow analysis model, we get flow diagram qualitatively combining with the normal data of the river, which could provide certain decision-making guidance to countries along the Zambezi River.

So, we recommend local countries removing the Kariba Dam and replace it with 11 dams along the Zambezi River and elevating water valve to storage water during February preparing for the dry weather and lower water valve to increase discharge in case of flood during August. Also, as for Malawi, Tanzania, and Zimbabwe, the three countries, covers less per capita water and lacks drinking water, so we suggest that they build more dam and increase water storage. 
All in all, the optimal scheme, shown in Fig. 7, is that we establish dams in point 1, point 2, point 3 , point 4 , point 5 , point 8 , point 9 , point 10 , point 11 , point 13 and point 14 and the minimum water storage of each dam node shown in Fig.6

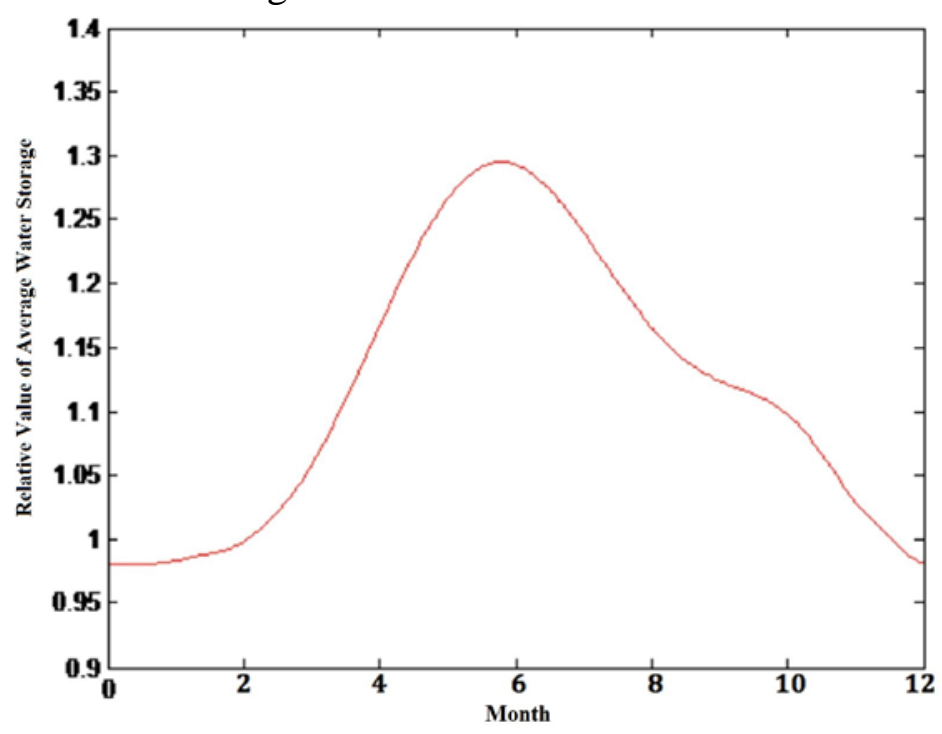

Fig. 7 the Curve Graph of Months

\section{References}

[1] Wikipedia, "Kariba Dam," Columbia Encyclopedia, 6th Ed. Retrieved 2007-07-31. https://en.wikipedia.org/wiki/Kariba_Dam

[2] Martin W Doyle. Emily H Stanley, etc, "Stream ecosystem response to small dam removal: Lessons from the Heartland,”Geomorphology, 2005(71):227--244.

[3] David McDermott Hughes, "Whites and Water: How Euro-Africans Made Nature at Kariba Dam” Journal of Southern African Studies Volume 32, Number 4, December 2006.

[4] Qiang Zhang, "Zambia per hydropower station computer monitoring system introduction,” Power plant automation,2015,(04):17-20.

[5] Lusaka. Chenje, Southern African Development Community and Zambezi River, Introductory Volume. Final Report. (Ed.) 2000.

[6] Xiaojuan Wang, Zhou Li, "Irrigation water use efficiency and influence factors analysis," China's rural economy, 2005,(07):11-18.

[7] Zhang Zhixiong,Guo Baozhu, "The Well-Posedness and Regularity of the Euler-Bernoulli Equation with Variable Coefficients, ” Chinese Academy of Science s, Beijing 100080.

[8] Lan Bai,Huichun Wang,Weiqing Zhen. "Feed water system pressure and flow in the relationship,” Industry and technology BBS,2016,(05):81-82

[9] Gerardo Guerra, "A Solid State Transformer model for power flow calculations Original Research Article ” International Journal of Electrical Power \& Energy Systems, Volume 89, July 2017, Pages 40-51. 